\title{
Brain Functional Alternations of the Pain-related Emotional and Cognitive Regions in Patients with Chronic Shoulder Pain
}

This article was published in the following Dove Press journal:

Journal of Pain Research

\author{
Jin-Ling $\mathrm{Li}^{1,2}$ \\ Chao-Qun Yan' \\ Xu Wang $\mathbb{D}^{3}$ \\ Shuai Zhang ${ }^{4}$ \\ $\mathrm{Na}$ Zhang $^{5}$ \\ Shang-Qing $\mathrm{Hu}^{\prime}$ \\ Li-Qiong Wang' \\ Cun-Zhi Liu'
}

'School of Acupuncture and Moxibustion, Beijing University of Chinese Medicine, Beijing, People's Republic of China; ${ }^{2}$ Department of Acupuncture and Moxibustion, Dongfang Hospital, Beijing University of Chinese Medicine, Beijing, People's Republic of China; ${ }^{3}$ School of Life Sciences, Beijing University of Chinese Medicine, Beijing, People's Republic of China; ${ }^{4}$ Department of Acupuncture and Moxibustion, Wangjing Hospital, Beijing, People's Republic of China; ${ }^{5}$ School of Acupuncture and Moxibustion, Shandong University of Chinese Medicine, Shandong, People's Republic of China
Correspondence: Cun-Zhi Liu School of Acupuncture and Moxibustion, Beijing University of Chinese Medicine, II Bei San Huan Dong Lu, Chaoyang District, Beijing 100029, People's Republic of China Tel +86 I590I26I692

Email Icz_tg@126.com
Objective: Chronic shoulder pain (CSP) is a common health problem associated with shoulder dysfunction and persistent pain for many different reasons. However, the studies of pain-related functional brain regions in CSP have been poorly investigated. The main purpose of our study was to observe whether there are abnormal functional changes in brain regions in patients with CSP by using functional magnetic resonance imaging (fMRI).

Patients and Methods: We compared the differences of brain regions between 37 patients with CSP and 24 healthy controls (HC) using regional homogeneity (ReHo) method. The patients with chronic shoulder pain and healthy controls were matched for age and gender. Brain regions which had abnormal ReHo values were defined as seed region of interests. The approach of seed-based functional connectivity (FC) was further performed to analyze the connectivity between the seeds and whole brain regions. The relationship between abnormal regions and current clinical pain was also evaluated.

Results: Compared to healthy controls, the patients with CSP showed increased ReHo values in the left middle temporal gyrus and decreased ReHo values in right orbitofrontal cortex (OFC). The seed-based analyses demonstrated decreased connectivity between the right $\mathrm{OFC}$ and right rectus, superior frontal gyrus in patients with chronic shoulder pain. However, a correlation between ReHo values and clinical characteristics in CSP patients was not found.

Conclusion: The observed results indicate that there are abnormal ReHo values in brain regions of patients with CSP, especially in the OFC and middle temporal gyrus. Our findings demonstrate that the experience of CSP patients may be mainly associated with cognitiveaffective pain processing, rather than nociception.

Keywords: functional magnetic resonance imaging, chronic shoulder pain, cognition, emotion

\section{Introduction}

Chronic shoulder pain (CSP) is a common musculoskeletal disorder associated with shoulder stiffness, a limited range of motion and persistent pain. ${ }^{1}$ In the general population, the prevalence and incidence of CSP were about $66.7 \%^{2}$ and $2.5 \%,{ }^{3}$ respectively. Patients with CSP need cost about $€ 4139$ per year ${ }^{4}$ and CSP causes about 4.5 million visits to physicians in the United States. ${ }^{5}$ CSP can lower the quality of life, ${ }^{6}$ reduce the ability to work, cause emotional disorders and has substantial socio-economic impact. ${ }^{7}$ For these reasons, it is appropriate to explore the neural mechanisms of CSP, which are associated with clinical treatment. 
Pain is an essentially subjective experience, associated with emotion and cognition which can be experienced differently by each individual and their personality. ${ }^{8}$ One systematic review had an interesting finding that our brain reserves certain activity for pain-related factors even in the resting state. ${ }^{9}$ The painrelated brain regions primarily included the thalamus, insula and anterior cingulate, while other brain regions were likely to be stimulus-specific. ${ }^{10}$ In recent years, many studies have valuated the underlying neurophysiological mechanism of brain functional changes in low back pain, ${ }^{11}$ trigeminal neuralgia, ${ }^{12}$ chronic migraine, ${ }^{13,14}$ fibromyalgia ${ }^{15}$ knee osteoarthritis, ${ }^{16}$ visceral pain, ${ }^{17}$ but literature focused on abnormal pattern of cerebral networks for pain processing in patients with shoulder pain is insufficient. In the brain, the functional interaction effects of pain, cognitive as well as negative affect have been widely reported ${ }^{18,19}$ and multiple mechanisms are needed to fully explain the emotional and cognitive brain alternations associated with pain. ${ }^{20}$ It is uncertain whether CSP patients have any changes in brain.

Resting-state functional magnetic resonance imaging (rs-fMRI) is a useful technique which can determine alternations of brain structure and function in patients with chronic pain. The blood-oxygen-level-dependent (BOLD) signals can measure the spontaneous and intrinsic neuronal activity as a better indicator of processing within an area, and also can be used to glean robust information about functional localization within the brain. ${ }^{21,22}$ Many studies use fMRI to reveal central pain processing mechanisms and provide an evidence for a musculoskeletal or neurologic disease. Chronic pain could impact brain function and behavior by altering the flow and integration of information between cerebral regions. ${ }^{23}$ Regional homogeneity ( $\mathrm{ReHo}$ ) is applied to demonstrate the regional consistency of spontaneous neuronal activity characteristics of each voxel within the whole brain. ${ }^{24}$ Moreover, among all kinds of rs-fMRI data analysis methods, the ReHo shows high test-retest reliability ${ }^{25}$ and superior sensitivity in detecting complex spontaneous hemodynamic responses in brain function. The alterations of ReHo can be used as neural markers to reveal the mechanisms of some diseases. ${ }^{26}$ Central system may play an important role in CSP, although the main pathophysiology changes of chronic shoulder pain remain unclear.

Overall, many neuroimaging studies have demonstrated that patients with pain-related symptoms have abnormal neuronal activity and structural function, ${ }^{11-17}$ few have focused on chronic shoulder pain. Therefore, the main objective of our study is to investigate how chronic shoulder pain affects brain function. In view of the insufficient studies on the brain mechanisms of CSP, we preliminarily made a hypothesis that patients with CSP would exhibit abnormal ReHo values in certain functional regions compared with healthy controls.

\section{Materials and Methods Subjects}

A total of 39 patients with CSP were recruited from department of acupuncture at Beijing Hospital Traditional Chinese Medicine Affiliated to Capital Medical University from December 2016 to July 2017. The study was conducted in accordance with the Declaration of Helsinki. All the participants were informed about the entire study procedures and provided written informed consent.

The inclusion criteria of CSP patients were: 1) age from 45 to 65 years; 2) right-handed; ${ }^{27,28} 3$ ) shoulder pain duration of at least 6 weeks, and not more than 24 months; 4) visual analogue scale (VAS) score between 50 and $100 \mathrm{~mm}$ (VAS score of $0-100 \mathrm{~mm}, 0 \mathrm{~mm}$ means "no pain" and $100 \mathrm{~mm}$ means "worst pain") in the past week. The exclusion criteria were: 1) other chronic pain conditions or shoulder trauma, such as headache, back pain, rheumatoid arthritis; 2) current therapy involving analgesics for the pain; 3) obvious abnormalities of brain structures on conventional MRI scan, such as tumors; 4) a history of neuropsychiatric disorders, such as depressive disorder, bipolar disorder, schizophrenias, stroke, or multiple sclerosis; 5) a history of alcohol or substance abuse; 6) had contraindications to have an MRI.

Healthy controls (HC) were recruited by using advertisements and they were age -and gender-matched to patients. The inclusion criteria for HC were as follows: 1) were righthanded; 2) no history of any chronic pain; 3) no organic lesion or altered brain structures on MRI; 4) no history of alcohol or medications abuse; 5) no neurological or psychiatric disorders; 6) no contraindications to have an MRI.

In our study, thirty-nine patients with chronic shoulder pain and twenty-six healthy controls fulfilled the inclusion criteria. Rs-fMRI data were collected from all participants and each participant had fully finished MRI scan. There were two CSP patients and two healthy controls who were excluded because of excessive head motion (head motion $>2 \mathrm{~mm}$ and $>2.0$ degree) during MRI scanning. Before MRI scanning, all CSP patients need to complete a VAS score and a Constant-Murley score (CMS) to evaluate the degree of their pain. 


\section{MRI Data Acquisition}

Functional MRI was carried out using a Siemens 3.0 Tesla scanner (Skyra, Siemens, Erlangen, Germany) equipped with a standard head coil. All participants underwent functional MRI scanning at the Department of Radiology for Beijing Hospital of Traditional Chinese Medicine Affiliated to Capital Medical University. Foam pads were used to minimize head motion and earplugs were used to reduce noise interference. During the rs-fMRI acquisition, participants were in a supine position with their eyes closed, and were instructed to remain awake, avoid thinking and keep still.

Rs-fMRI data were obtained using a gradient echo planar imaging (EPI) sequence with the following parameters: whole brain, repetition time $(\mathrm{TR})=2000 \mathrm{~ms}$, echo time $(\mathrm{TE})=30 \mathrm{~ms}$, field of view $(\mathrm{FOV})=220 \mathrm{~mm} \times$ $220 \mathrm{~mm}$, flip angle $(\mathrm{FA})=90^{\circ}$, slice thickness $/$ gap $=3.5 /$ $0.6 \mathrm{~mm}$, axial slices $=33$, in-plane resolution $=64 \times 64$, and 240 volumes. All participants reported that they remained awake during the entire scanning time.

\section{Imaging Data Preprocessing}

The functional data were preprocessed using statistical parameter mapping SPM8 (http://www.fil.ion.ucl.ac.uk/ $\mathrm{spm} /$ software/spm8) for MATLAB and the Data Processing Assistant and Resting-State fMRI (DPARSF) toolbox. ${ }^{29}$ In order to get optimal and balanced rs-fMRI data, the first 10 volumes were removed from each participant. The remaining volumes were corrected using slice timing, spatially realigned and head motion. A participant was to be removed from the analysis if head motion was more than $2 \mathrm{~mm}$ in any direction. The corrected data were spatially normalized to fit a standard brain template in the Montreal Neurological Institute (MNI) coordinate space and then re-sampled to $3 \times 3 \times 3 \mathrm{~mm}^{3}$. The linear trend of the signals was removed over each run, and the nuisance signals (white matter signals, cerebral spinal fluid signal, and Friston 24 head motion) parameters were regressed. Subsequently, a band pass filter with a frequency range of $0.01-0.08 \mathrm{~Hz}$ was applied, and these images were smoothed with an $8 \mathrm{~mm}$ full-width half-maximum Gaussian kernel.

\section{ReHo Analysis}

ReHo was computed using the Kendall concordance coefficient with the DPARSF toolbox. Kendall harmony coefficient is used to demonstrate the brain function regions to be at a different time sequence consistency of voxel. The whole brain ReHo maps were acquired by computing the consistency of the time series between each voxel and its adjacent 26 voxel in brain. ${ }^{12}$ The cluster-level corrected for Gaussian Random Field theory correction (GRF) (voxel level $\mathrm{p}<0.001$ and cluster level $\mathrm{p}<0.05$, TwoTailed) was applied for the between-groups analysis.

\section{Functional Connectivity Analysis}

A seed-based analysis was applied to measure functional connectivity by extracting time series from the region of interest (ROI). The seed region was defined as a specific area which was identified by ReHo analysis between the CSP patients and healthy controls using a temporal correlation. ${ }^{30}$ Seed-based correlation was calculated between the seed and whole-brain voxels by Pearson's correlation analysis. The obtained FC maps were converted to $\mathrm{z}$ values using Fisher's r-to-z transformation to normalized connectivity map of each individual. The cluster level corrected for Gaussian Random Field theory correction (GRF) (voxel level $p<0.01$ and cluster level $p<0.05$, Two-Tailed) was applied for the between-groups analysis.

\section{Statistical Analysis}

Demographic and clinical data obtained from participants were analyzed by the SPSS 22 software. Shapiro-Wilk test was used to detect the normal distribution. Two-sample independent $t$-test and Chi-square test were used to assess the differences in baseline age and gender. A two-sample $t$-test was performed to analyzed ReHo maps and FC maps comparing CSP patients and healthy controls using the DPARSF software. Cluster level corrected for multiple comparisons (GRF) was applied to the ReHo and FC analysis. Pearson's correlation coefficient was implemented to verify the relationships between abnormal ReHo values or seed-based FC values and clinical characteristics in patients, including VSA, CMS and shoulder pain duration.

\section{Results}

\section{Demographic and Clinical Characteristics}

Table 1 shows details about demographic and clinical characteristics of participants. Thirty-seven patients (mean age: $53.65 \pm 5.45$ years, 17 females) and 24 matched HCs (mean age: $55.83 \pm 4.72$ years, 14 females) completed the entire study. And the mean duration of CSP was 6.38 \pm 6.05 months, the mean pain score on the visual analogue 
Table I Demographic and Clinical Characteristics of CSP Patients and Healthy Controls

\begin{tabular}{|l|l|l|l|}
\hline Parameter & $\begin{array}{l}\text { CSP } \\
\text { Patients }\end{array}$ & $\begin{array}{l}\text { Healthy } \\
\text { Controls }\end{array}$ & P-value \\
\hline Age(year) & $53.65 \pm 5.45$ & $55.83 \pm 4.72$ & 0.113 \\
\hline $\begin{array}{l}\text { Sex } \\
\text { Male }\end{array}$ & 20 & 10 & \\
Female & 17 & 14 & 0.344 \\
\hline Pain duration(month) & $6.38 \pm 6.05$ & N/A & \\
Pain degree (VAS) & $66.35 \pm 13.32$ & N/A & \\
Shoulder function (CMS) & $53.24 \pm 13.84$ & N/A & \\
\hline
\end{tabular}

Note: Data are mean \pm standard deviation for age, pain duration, pain degree and shoulder function.

Abbreviation: N/A, not applicable.

scale (VAS) was $66.35 \pm 13.32$, and the mean shoulder function score on CMS was 53.24 \pm 13.84 in patients.

\section{ReHo Differences Between Groups}

After correction for GRF, the ReHo values in the CSP group were significantly increased in left middle temporal gyrus and decreased in right orbitofrontal cortex (OFC) compared with healthy controls (Figure 1 and Table 2). No correlation was found between ReHo values in abnormal brain regions and clinical characteristics.

\section{Seed-Based Functional Connectivity Between Groups}

Due to the significantly different ReHo maps in left middle temporal gyrus and right orbitofrontal cortex between CSP and $\mathrm{HC}$, we chose the left middle temporal gyrus and right orbitofrontal cortex as seed regions and performed a whole brain FC analysis. Compared to HC, the results of seedbased resting-state functional connectivity analysis shown decreased FC between OFC and right rectus, superior frontal gyrus in patients (Figure 2 and Table 2). However, no brain regions with connectivity were found in left middle temporal gyrus.

\section{Discussion}

Our study aimed to explore the abnormal functional brain regions that are associated with pain during the resting state in patients with CSP. The primary findings showed that patients with CSP had significant increased ReHo values in middle temporal gyrus and decreased ReHo values in orbitofrontal cortex (OFC), compared to healthy controls. Two specific brain regions are both involved in pain regulation, related with emotion, memory and cognition. $^{31-33}$ Further seed-based FC analysis revealed decreased FC between OFC and right rectus as well as superior frontal gyrus, which are important regions related to cognition and the experience of pain. ${ }^{34}$

Pain causes physical discomfort and psychological affective reactions ${ }^{32}$ and is perceived during consciousness. ${ }^{35}$ OFC plays an important role in the cognitive processing of pain perception, for example, it can ease pain through adjusting and controlling endogenous opioid release. ${ }^{36}$ Multivariate models are used to analyze brain responses to pain in fMRI studies, and OFC has been found to be a crucial region which could precisely distinguish pain and non-pain experience. ${ }^{31}$ The OFC has the capacity for pain and emotional regulation. Negative affectivity is associated with painrelated cognitions and OFC mediates automatic emotion regulation. $^{32}$ Spontaneous magnetoencephalography (MEG) suggested that patients with fibromyalgia (FM) had abnormal spectral powers in OFC related to emotional and cognitive modulation of pain. ${ }^{37}$ Another study indicated that deactivation of OFC was associated with nociceptive stimulation and sympathetic activation, ${ }^{38}$ and the similar deactivated OFC was also observed in our study. Chronic pain may modify the functional patterns of OFC, and the deactivation of OFC also contributes to pain perception. Comparing CSP patients to healthy controls, our findings of altered ReHo values in OFC may be one reason for pain persistence in chronic shoulder pain.

During our study, hypoactive OFC was observed in chronic shoulder pain, and inhibitory compensation can be used to explain this finding, to balance the overexcitation input in pain modulation for long-term pain condition. ${ }^{39}$ Chronic pain is a complex mechanism that affects our emotions and cognition; it also could distort our judgment and prediction based on emotional guide. ${ }^{40} \mathrm{OFC}$ is an important subdivision of prefrontal cortex (PFC) critically involved in subjective decision-making. ${ }^{41,42}$ Behavioral decision-making in adolescents can be adversely affected due to deactivation in $\mathrm{OFC}^{43}$ and animal experiment further confirmed that the synchronous expression of inactive OFC and active NAC (Nucleus accumbens) can undermine behavioral control. ${ }^{44}$ Our study reveals obvious difference in OFC, and also provide additional evidence for decision-making impairment in chronic pain conditions. 

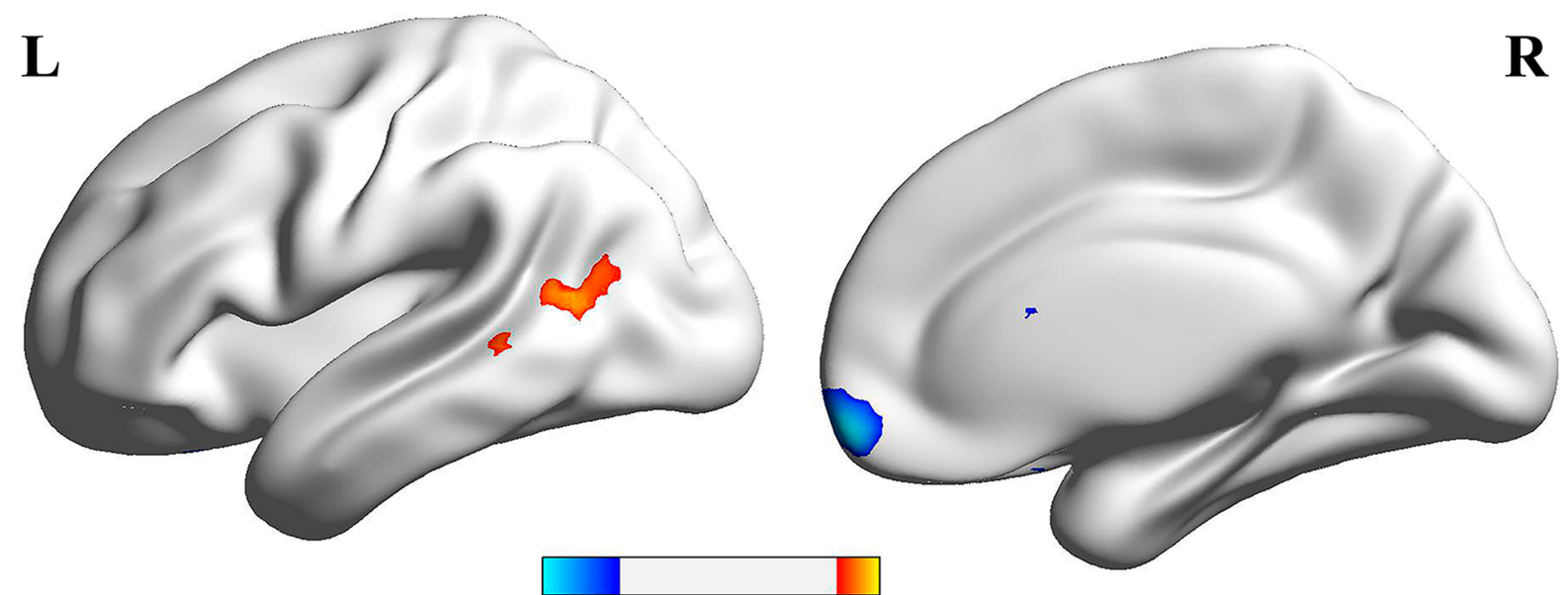

$\mathbf{R}$

$-5.97$

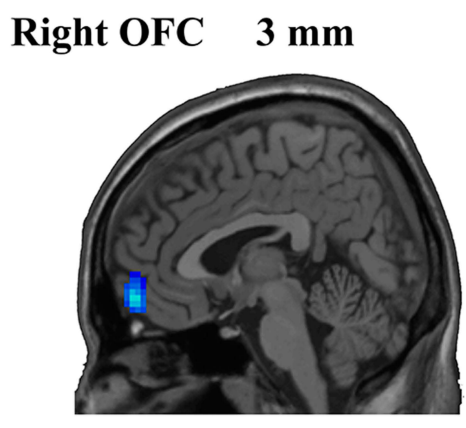

Left MTG 6 mm

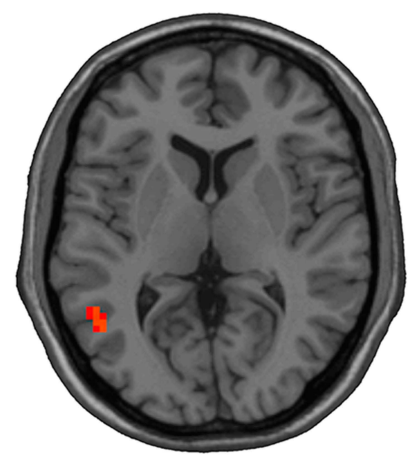

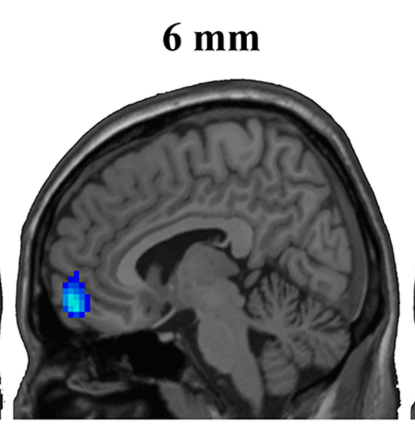

$10 \mathrm{~mm}$

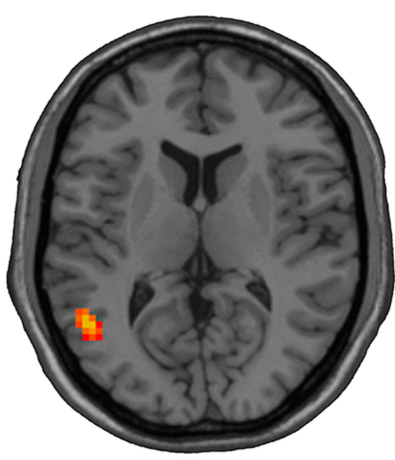

4.85
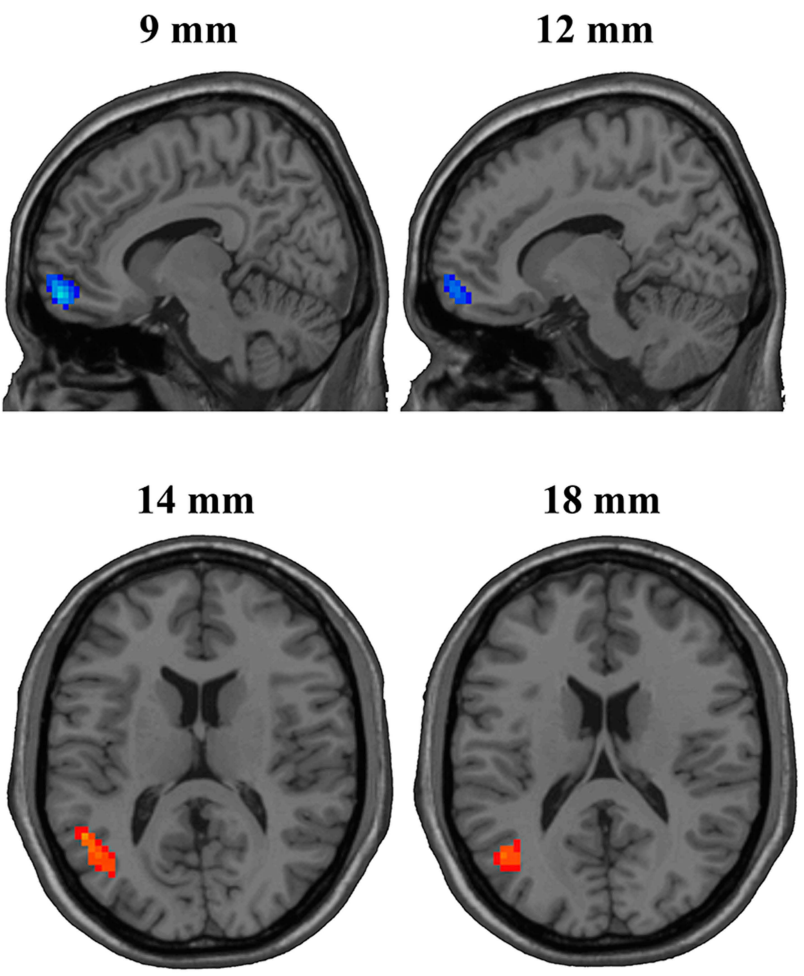

Figure I Medial views and axial medial views of significant changes in ReHo between chronic shoulder pain (CSP) group and healthy controls (HC) group. The hot color indicates that chronic shoulder pain (CSP) group had increased ReHo compared with healthy controls (HC) group. The cool color indicates that chronic shoulder pain (CSP) group had decreased ReHo compared with healthy controls $(\mathrm{HC})$ group. Gaussian Random Field theory correction (GRF) correction with significant voxel level $\mathrm{p}<0.0 \mathrm{I}$ and cluster level $p<0.05$, Two-Tailed.

In this study, seed-based FC approach showed decreased connectivity between $\mathrm{OFC}$ and right rectus gyrus and superior frontal gyrus in patients. Based on current studies, the rectus gyrus is considered to be linked with the recovery of consciousness ${ }^{45,46}$ and dynamical emotion processing. ${ }^{47}$ Superior frontal gyrus has similar functions as $\mathrm{OFC}$ and middle temporal gyrus, which also is an important region related to cognition, social learning and painful experience. ${ }^{34}$ A metaanalysis demonstrated that patients with Alzheimer's disease and mid-cognitive impairment had compensatory activity in superior frontal gyrus and medial temporal gyrus. ${ }^{48}$ One study demonstrated that the worsening pain symptoms may attribute to the thinner cortex in 
Table 2 Brain Regions Showing ReHo and Seed-Based Functional Connectivity Differences Between Two Groups

\begin{tabular}{|c|c|c|c|c|c|c|}
\hline \multirow[t]{2}{*}{$\begin{array}{l}\text { Brain } \\
\text { Regions }\end{array}$} & \multirow[t]{2}{*}{ Side } & \multicolumn{3}{|c|}{$\begin{array}{l}\text { MNI } \\
\text { Coordinates }\end{array}$} & \multirow{2}{*}{$\begin{array}{l}\begin{array}{l}\text { Cluster } \\
\text { Size }\end{array} \\
\text { (mm3) }\end{array}$} & \multirow[t]{2}{*}{$\begin{array}{l}\text { Peak } \\
\text { t value }\end{array}$} \\
\hline & & $\mathbf{x}$ & y & $\mathbf{z}$ & & \\
\hline \multicolumn{7}{|c|}{ ReHo differences, CSP patient $<$ healthy controls } \\
\hline $\begin{array}{l}\text { Orbitofrontal } \\
\text { Cortex }\end{array}$ & Right & 6 & 60 & -12 & 150 & -5.97 \\
\hline \multicolumn{7}{|c|}{ ReHo differences, CSP patient>healthy controls } \\
\hline $\begin{array}{l}\text { Middle } \\
\text { Temporal } \\
\text { Gyrus }\end{array}$ & Left & -51 & -57 & 12 & 85 & 4.73 \\
\hline \multicolumn{7}{|c|}{$\begin{array}{l}\text { Seed-based functional connectivity: the right OFC, CSP } \\
\text { patients<healthy controls }\end{array}$} \\
\hline $\begin{array}{l}\text { Rectus } \\
\text { Superior } \\
\text { Frontal Gyrus }\end{array}$ & $\begin{array}{l}\text { Right } \\
\text { Right }\end{array}$ & 9 & 12 & -12 & 327 & -5.30 \\
\hline
\end{tabular}

superior frontal gyrus and anterior cingulated in cervical spondylosis patients. ${ }^{49}$ And pain could lead to lower connectivity between precentral gyrus and superior frontal gyrus which participate in the processing of painrelated emotional and cognition in classical trigeminal neuralgia. ${ }^{50}$ It is similar to our findings.

Another important finding in our study was the hyperactive middle temporal gyrus in CSP patients compared to healthy controls. Since many functional regions are shared for pain processing and cognitive processing, patients with chronic pain often complain cognitive impairment and inattention. ${ }^{51,52}$ Pain-related attention and emotion are closely associated with medial temporal cortex, which is considered to be linked with pain integration and pain perception. ${ }^{33}$ Increased grey matter volume (GMV) in the middle temporal lobes was found in postherpetic neuralgia ${ }^{53}$ and low-back pain altered several functional brain regions including middle temporal lobes, ${ }^{54,55}$ and a similar result was reported in burning mouth syndrome patients. ${ }^{54}$ Our finding also demonstrated that the middle temporal gyrus engages in pain regulation. A study of fibromyalgia reported that dampened deactivation in the medial temporal lobe may contribute to the prolongation of pain sensation. ${ }^{56}$ A Hyperactive medial temporal lobe, especially the anterior temporal pole was considered as a biomarker for migraine attacks. ${ }^{57} \mathrm{~A}$ similar, activated middle temporal lobe was observed in our study, which indicates the middle temporal lobe is associated with pain perception in CSP. Kucyi et al found enhanced functional coupling between PAG and default mode network regions (involved medial temporal lobe, mPFC and PCC) linked with attention on pain and considered the pain-related cognitive processing as a dynamic and continuous procedure. ${ }^{58}$ In our study, the activated middle temporal lobe may be responsible for more attention to pain in CSP patients.

There are some limitations in this study. First, there are many distinctions between sub-categories of shoulder pain due to ageing, somatization tendency, mental health and occupation. We did not distinguish the functional changes between different shoulder pain categories. ${ }^{59}$ Second, left and right shoulder pain have not been discussed separately, we will perform additional analyses to compare whether there is any differences between left and right shoulder pain in cerebral regions in future studies. Third, patients with CSP generally have anxiety or depression, ${ }^{60}$ but emotion-related scales were not investigated in this study. We should assess patients' emotion condition to verify what role pain-related anxiety or depression plays in shoulder pain. Forth, participants with age range between 45 and 65 years old were recruited which may restrict the generalization of the present results to other populations. Future studies with a wider age range are needed to increase external validity.

\section{Conclusion}

In summary, our findings illustrate CSP patients display abnormal ReHo values in OFC and middle temporal cortex compared with healthy controls, and decreased FC between the $\mathrm{OFC}$ and rectus gyrus as well as superior frontal gyrus. Given the impact of pain on emotion, cognitive and attention abilities, the current study provides evidence for cognitive-affective pain modulation and perception processing associated with chronic shoulder pain. The results also support that the abnormal ReHo values in the OFC and middle temporal cortex are involved in the mechanism of chronic shoulder pain.

\section{Ethics and Consent Statement}

The study was approved by the Research Ethical Committee of Beijing Hospital Traditional Chinese Medicine Affiliated 


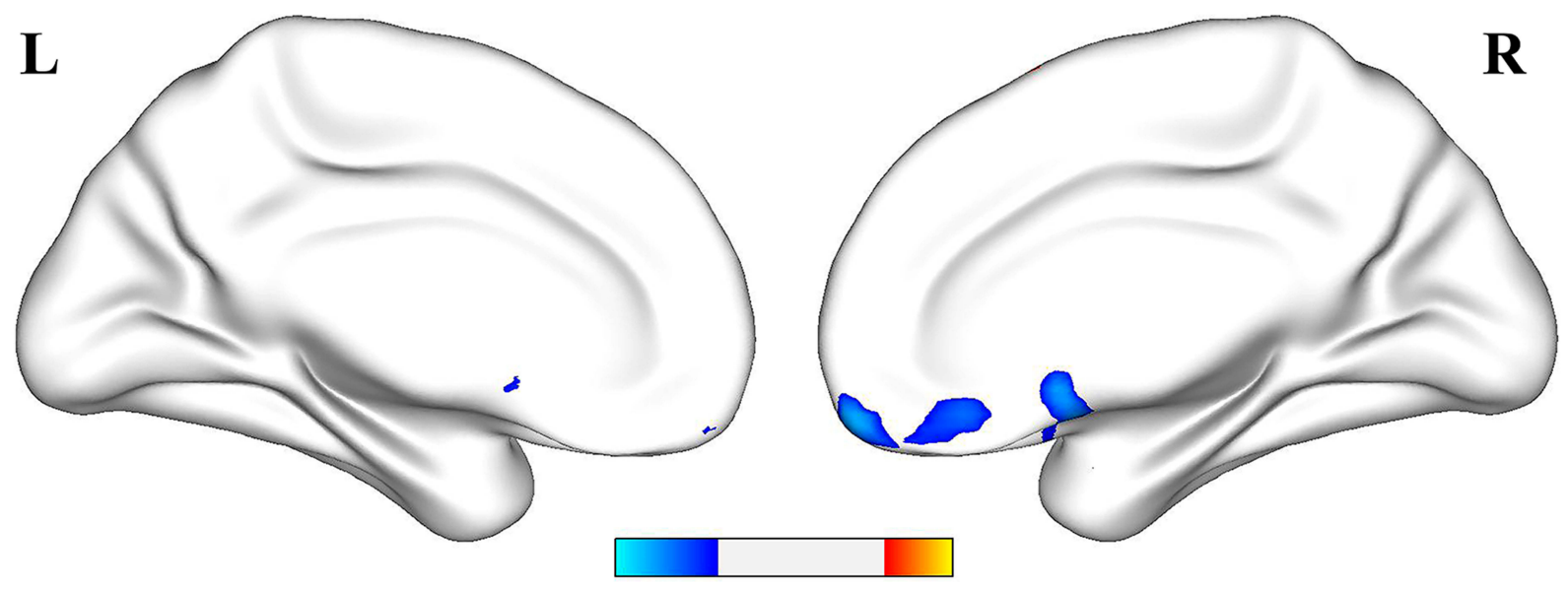

$-5.97$

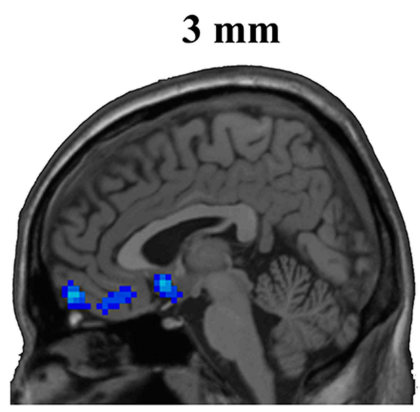

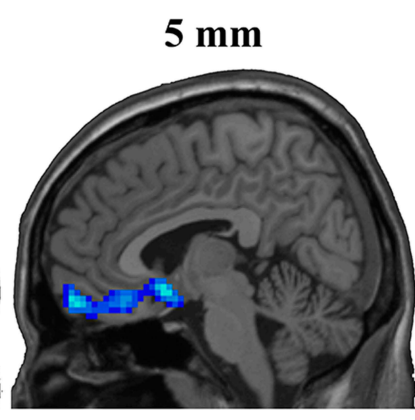
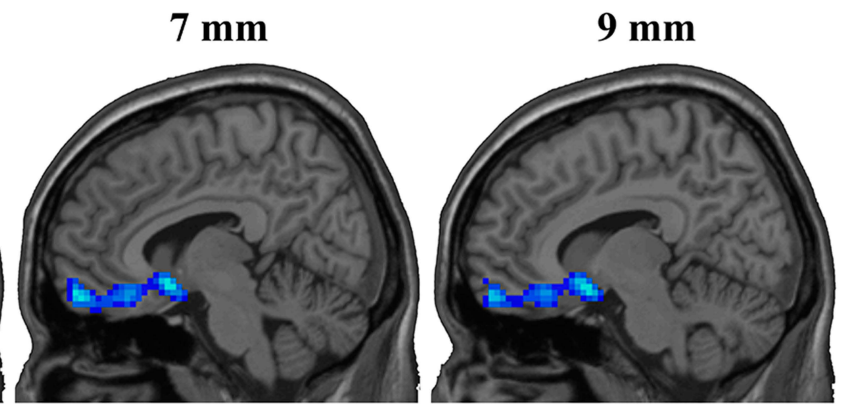

Figure 2 Group differences in seed-based functional connectivity. The seed was defined as the right orbitofrontal cortex. The cool color indicated decreased functional connectivity in the chronic shoulder pain (CSP) group compared with healthy controls (HC) group. Gaussian Random Field theory correction (GRF) correction with significant voxel level $p<0.01$ and cluster level $p<0.05$, Two-Tailed.

to Capital Medical University (201315). We obtained written informed consent from all participants.

\section{Acknowledgments}

The authors thank all the participants in this study. This work was supported by the Beijing Technology Development of Traditional Chinese Medicine Foundation-Annual Planning Project (JJ 2013-40).

\section{Author Contributions}

Cun-Zhi Liu conceived and directed the study design, Shuai Zhang, Chao-Qun Yan, Shang-Qing Hu participated in study design and performed the experiments, $\mathrm{Na}$ Zhang, Jin-Ling $\mathrm{Li}$ and $\mathrm{Xu}$ Wang analyzed and interpreted the data, Jin-Ling Li, Chao-Qun Yan, Na Zhang, Shuai Zhang and Shang-Qing Hu drafted the manuscript. $\mathrm{Xu}$ Wang, Li-Qiong Wang and Cun-Zhi Liu revised the manuscript. Chao-Qun Yan and Li-Qiong Wang assisted in imaging analysis. All authors contributed to data analysis, drafting or revising the article, gave final approval of the version to be published, and agree to be accountable for all aspects of the work. And if you need the original data, please contact Cun-Zhi Liu, email: lcz_tg@126.com.

\section{Disclosure}

All authors declare no conflicts of interest in this work.

\section{References}

1. Cools AM, Michener LA. Shoulder pain: can one label satisfy everyone and everything? Br J Sports Med. 2017;51(5):416-417. doi:10.1136/bjsports-2016-096772

2. Luime JJ, Koes BW, Hendriksen IJ, et al. Prevalence and incidence of shoulder pain in the general population; a systematic review. Scand J Rheumatol. 2004;33(2):73-81. doi:10.1080/03009740310004667

3. Luime JJ, Koes BW, Miedem HS, Verhaar JA, Burdorf A. High incidence and recurrence of shoulder and neck pain in nursing home employees was demonstrated during a 2-year follow-up. $J$ Clin Epidemiol. 2005;58(4):407-413. doi:10.1016/j.jclinepi.2004.01.022

4. Virta L, Joranger P, Brox JI, Eriksson R. Costs of shoulder pain and resource use in primary health care: a cost-of-illness study in Sweden. BMC Musculoskelet Disord. 2012;13(1):17. doi:10.1186/1471-247413-17 
5. Oh LS, Wolf BR, Hall MP, Levy BA, Marx RG. Indications for rotator cuff repair: a systematic review. Clin Orthop Relat Res. 2007;455:52-63. doi:10.1097/BLO.0b013e31802fc175

6. Herin F, Vezina M, Thaon I, Soulat JM, Paris C. Predictors of chronic shoulder pain after 5 years in a working population. Pain. 2012;153 (11):2253-2259. doi:10.1016/j.pain.2012.07.024

7. Urwin M, Symmons D, Allison T, et al. Estimating the burden of musculoskeletal disorders in the community: the comparative prevalence of symptoms at different anatomical sites, and the relation to social deprivation. Ann Rheum Dis. 1998;57(11):649-655. doi:10.11 36/ard.57.11.649

8. Ploner M, Lee MC, Wiech K, Bingel U, Tracey I. Prestimulus functional connectivity determines pain perception in humans. Proc Natl Acad Sci U S A. 2010;107(1):355-360. doi:10.1073/pnas.0906 186106

9. Malfliet A, Coppieters I, Van Wilgen P, et al. Brain changes associated with cognitive and emotional factors in chronic pain: a systematic review. Eur J Pain. 2017;21(5):769-786. doi:10.1002/ ejp.2017.21.issue-5

10. Duerden EG, Albanese M-C. Localization of pain-related brain activation: a meta-analysis of neuroimaging data. Hum Brain Mapp. 2013;34(1):109-149. doi:10.1002/hbm.v34.1

11. Letzen JE, Robinson ME. Negative mood influences default mode network functional connectivity in patients with chronic low back pain: implications for functional neuroimaging biomarkers. Pain. 2017;158(1):48-57. doi:10.1097/j.pain.0000000000000708

12. Xiang CQ, Liu WF, Xu QH, et al. Altered spontaneous brain activity in patients with classical trigeminal neuralgia using regional homogeneity: a resting-state functional MRI Study. Pain Pract. 2019;19 (4):397-406.

13. Kisler LB, Granovsky Y, Coghill RC, et al. Do patients with interictal migraine modulate pain differently from healthy controls? A psychophysical and brain imaging study. Pain. 2018;159 (12):2667-2677. doi:10.1097/j.pain.0000000000001380

14. Androulakis XM, Krebs KA, Jenkins C, et al. Central executive and default mode network intranet work functional connectivity patterns in chronic migraine. J Neurol Disord. 2018;6(5). doi:10.4172/23296895.1000393

15. Ichesco E, Schmidt-Wilcke T, Bhavsar R, et al. Altered resting state connectivity of the insular cortex in individuals with fibromyalgia. J Pain. 2014;15(8):815-826. doi:10.1016/j.jpain.2014.04.007

16. Pujol J, Martinez-Vilavella G, Llorente-Onaindia J, et al. Brain imaging of pain sensitization in patients with knee osteoarthritis. Pain. 2017;158(9):1831-1838. doi:10.1097/j.pain.0000000000000985

17. Koenen LR, Icenhour A, Forkmann K, et al. Greater fear of visceral pain contributes to differences between visceral and somatic pain in healthy women. Pain. 2017;158(8):1599-1608. doi:10.1097/j.pain.00 00000000000924

18. Coen SJ, Yaguez L, Aziz Q, et al. Negative mood affects brain processing of visceral sensation. Gastroenterology. 2009;137 (1):253-261, 261. doi:10.1053/j.gastro.2009.02.052

19. Shackman AJ, Salomons TV, Slagter HA, Fox AS, Winter JJ, Davidson RJ. The integration of negative affect, pain and cognitive control in the cingulate cortex. Nat Rev Neurosci. 2011;12 (3):154-167. doi:10.1038/nrn2994

20. Kragel PA, Kano M, Van Oudenhove L, et al. Generalizable representations of pain, cognitive control, and negative emotion in medial frontal cortex. Nat Neurosci. 2018;21(2):283-289. doi:10.1038/ s41593-017-0051-7

21. Baria AT, Baliki MN, Parrish T, Apkarian AV. Anatomical and functional assemblies of brain BOLD oscillations. J Neurosci. 2011;31 (21):7910-7919. doi:10.1523/JNEUROSCI.1296-11.2011

22. Hall CN, Howarth C, Kurth-Nelson Z, Mishra A. Interpreting BOLD: towards a dialogue between cognitive and cellular neuroscience. Philos Trans R Soc Lond B Biol Sci. 2016;371(1705):20150348. doi:10.1098/rstb.2015.0348
23. Baliki MN, Baria AT, Apkarian AV. The cortical rhythms of chronic back pain. J Neurosci. 2011;31(39):13981-13990. doi:10.1523/ JNEUROSCI.1984-11.2011

24. Zuo XN, Di Martino A, Kelly C, et al. The oscillating brain: complex and reliable. Neuroimage. 2010;49(2):1432-1445. doi:10.1016/j. neuroimage.2009.09.037

25. Zuo XN, Xu T, Jiang L, et al. Toward reliable characterization of functional homogeneity in the human brain: preprocessing, scan duration, imaging resolution and computational space. Neuroimage. 2013;65:374-386. doi:10.1016/j.neuroimage.2012.10.017

26. Wu QZ, Li DM, Kuang WH, et al. Abnormal regional spontaneous neural activity in treatment-refractory depression revealed by resting-state fMRI. Hum Brain Mapp. 2011;32(8):1290-1299. doi:10. 1002/hbm. 21108

27. Shine JP, Valdés-Herrera JP, Tempelmann C, Wolbers T. Evidence for allocentric boundary and goal direction information in the human entorhinal cortex and subiculum. Nat Commun. 2019;10(1):4004. doi:10.1038/s41467-019-11802-9

28. Nair S, Nenert RE, Allendorfer JB, et al. Sex, age, and handedness modulate the neural correlates of active learning. Front Neurosci. 2019;13:961. doi:10.3389/fnins.2019.00961

29. Chao-Gan Y, Yu-Feng Z. DPARSF: a MATLAB toolbox for "pipeline" data analysis of resting-state fMRI. Front Syst Neurosci. 2010;4:13. doi:10.3389/fnsys.2010.00009

30. Fox MD, Snyder AZ, Vincent JL, Corbetta M, Van Essen DC, Raichle ME. The human brain is intrinsically organized into dynamic, anticorrelated functional networks. Proc Natl Acad Sci U S A. 2005;102(27):9673-9678. doi:10.1073/pnas.0504136102

31. Brodersen KH, Wiech K, Lomakina EI, et al. Decoding the perception of pain from fMRI using multivariate pattern analysis. Neuroimage. 2012;63(3):1162-1170. doi:10.1016/j.neuroimage.2012. 08.035

32. Perl ER. Pain mechanisms: a commentary on concepts and issues. Prog Neurobiol. 2011;94(1):20-38. doi:10.1016/j.pneurobio.2011. 03.001

33. Ploner M, Lee MC, Wiech K, Bingel U, Tracey I. Flexible cerebral connectivity patterns subserve contextual modulations of pain. Cereb Cortex. 2011;21(3):719-726. doi:10.1093/cercor/bhq146

34. Fan YT, Chen C, Cheng Y. The neural mechanisms of social learning from fleeting experience with pain. Front Behav Neurosci. 2016;10:11. doi:10.3389/fnbeh.2016.00011

35. Coghill RC, Talbot JD, Evans AC, et al. Distributed processing of pain and vibration by the human brain. J Neurosci. 1994;14 (7):4095-4108. doi:10.1523/JNEUROSCI.14-07-04095.1994

36. Dougherty DD, Kong J, Webb M, Bonab AA, Fischman AJ, Gollub RL. A combined [11C]diprenorphine PET study and fMRI study of acupuncture analgesia. Behav Brain Res. 2008;193 (1):63-68. doi:10.1016/j.bbr.2008.04.020

37. Lim M, Kim JS, Kim DJ, Chung CK. Increased low- and high-frequency oscillatory activity in the prefrontal cortex of fibromyalgia patients. Front Hum Neurosci. 2016;10:111. doi:10.3389/ fnhum.2016.00111

38. Maihofner C, Seifert F, Decol R. Activation of central sympathetic networks during innocuous and noxious somatosensory stimulation. Neuroimage. 2011;55(1):216-224. doi:10.1016/j.neuroimage.2010. 11.061

39. Iadarola MJ, Max MB, Berman KF, et al. Unilateral decrease in thalamic activity observed with positron emission tomography in patients with chronic neuropathic pain. Pain. 1995;63(1):55-64. doi:10.1016/0304-3959(95)00015-K

40. Seymour B, Dolan R. Emotion, decision making, and the amygdala. Neuron. 2008;58(5):662-671. doi:10.1016/j.neuron.2008.05.020

41. Murray EA, Rudebeck PH. Specializations for reward-guided decision-making in the primate ventral prefrontal cortex. Nat Rev Neurosci. 2018;19(7):404-417. doi:10.1038/s41583-018-0013-4 
42. Rich EL, Wallis JD. Decoding subjective decisions from orbitofrontal cortex. Nat Neurosci. 2016;19(7):973-980. doi:10.1038/nn.4320

43. Galvan A, Hare TA, Parra CE, et al. Earlier development of the accumbens relative to orbitofrontal cortex might underlie risk-taking behavior in adolescents. $J$ Neurosci. 2006;26 (25):6885-6892. doi:10.1523/JNEUROSCI.1062-06.2006

44. Meyer HC, Bucci DJ. Imbalanced activity in the orbitofrontal cortex and nucleus accumbens impairs behavioral inhibition. Curr Biol. 2016;26(20):2834-2839. doi:10.1016/j.cub.2016.08.034

45. Bai F, Shi Y, Yuan Y, et al. Altered self-referential network in resting-state amnestic type mild cognitive impairment. Cortex 2012;48(5):604-613. doi:10.1016/j.cortex.2011.02.011

46. Zhang L, Luo L, Zhou Z, et al. Functional connectivity of anterior insula predicts recovery of patients with disorders of consciousness. Front Neurol. 2018;9:1024. doi:10.3389/fneur.2018.01024

47. Melchers M, Markett S, Montag C, et al. Reality TV and vicarious embarrassment: an fMRI study. Neuroimage. 2015;109:109-117. doi:10.1016/j.neuroimage.2015.01.022

48. Browndyke JN, Giovanello K, Petrella J, et al. Phenotypic regional functional imaging patterns during memory encoding in mild cognitive impairment and alzheimer's disease. Alzheimers Dement. 2013;9 (3):284-294. doi:10.1016/j.jalz.2011.12.006

49. Woodworth DC, Holly LT, Mayer EA, Salamon N, Ellingson BM. Alterations in cortical thickness and subcortical volume are associated with neurological symptoms and neck pain in patients with cervical spondylosis. Neurosurgery. 2019;84(3):588-598. doi:10.1093/neuros/ nyy066

50. Tsai YH, Yuan R, Patel D, et al. Altered structure and functional connection in patients with classical trigeminal neuralgia. Hum Brain Mapp. 2018;39(2):609-621. doi:10.1002/hbm.23696

51. Ferreira KS, Oliver GZ, Thomaz DC, Teixeira CT, Foss MP. Cognitive deficits in chronic pain patients, in a brief screening test, are independent of comorbidities and medication use. Arq Neuropsiquiatr. 2016;74 (5):361-366. doi:10.1590/0004-282X20160071
52. Schiltenwolf M, Akbar M, Hug A, et al. Evidence of specific cognitive deficits in patients with chronic low back pain under long-term substitution treatment of opioids. Pain Physician. 2014; 17(1):9-20.

53. Cao S, Qin B, Zhang Y, et al. Herpes zoster chronification to postherpetic neuralgia induces brain activity and grey matter volume change. Am J Transl Res. 2018;10(1):184-199.

54. Lee YC, Jahng GH, Ryu CW, Byun JY. Change in gray matter volume and cerebral blood flow in patients with burning mouth syndrome. J Oral Pathol Med. 2019. doi:10.1111/jop.12838

55. Zhang SS, Wu W, Yang JM, Wang CH. Abnormal spontaneous brain activity in acute low-back pain revealed by resting-state functional MRI. Am J Phys Med Rehabil. 2017;96(4):253-259. doi:10.1097/ PHM.0000000000000597

56. Schreiber KL, Loggia ML, Kim J, Cahalan CM, Napadow V, Edwards RR. Painful after-sensations in fibromyalgia are linked to catastrophizing and differences in brain response in the medial temporal lobe. J Pain. 2017;18(7):855-867. doi:10.1016/j.jpain.2017. 02.437

57. Moulton EA, Becerra L, Maleki N, et al. Painful heat reveals hyperexcitability of the temporal pole in interictal and ictal migraine states. Cereb Cortex. 2011;21(2):435-448. doi:10.1093/cercor/bhq109

58. Kucyi A, Salomons TV, Davis KD. Mind wandering away from pain dynamically engages antinociceptive and default mode brain networks. Proc Natl Acad Sci U S A. 2013;110(46):18692-18697. doi:10.1073/pnas.1312902110

59. Sarquis LM, Coggon D, Ntani G, et al. Classification of neck/ shoulder pain in epidemiological research: a comparison of personal and occupational characteristics, disability, and prognosis among 12,195 workers from 18 countries. Pain. 2016;157(5):1028-1036. doi:10.1097/j.pain.0000000000000477

60. Toprak M, Erden M. Sleep quality, pain, anxiety, depression and quality of life in patients with frozen shoulder. $J$ Back Musculoskelet Rehabil. 2019;32(2):287-291. doi:10.3233/BMR171010
Journal of Pain Research

\section{Publish your work in this journal}

The Journal of Pain Research is an international, peer reviewed, open access, online journal that welcomes laboratory and clinical findings in the fields of pain research and the prevention and management of pain. Original research, reviews, symposium reports, hypothesis formation and commentaries are all considered for publication. The manuscript management system is completely online and includes a very quick and fair peer-review system, which is all easy to use. Visit http:// www.dovepress.com/testimonials.php to read real quotes from published authors. 